\title{
Estado del arte y retos para la mejora de sistemas de preparación en almacenes-Estudio Delphi
}

\author{
State of the art and challenges for picking systems improvement \\ in warehouse-a Delphi Study
}

\author{
Ander Errasti, Claudia Chackelson, Mikel Arcelus \\ Departamento de Organización Industrial,TECNUN - Escuela Superior de Ingenieros de la Universidad de Navarra \\ aerrasti@ceit.es cchackelson@tecnum.es
}

Fecha de recepción: 06-11-09

Fecha de aceptación: 03-03-10

Resumen: La preparación de pedidos o picking es un proceso clave dentro de la gestión de almacenes por su impacto sobre los costes operativos y por la alta repercusión en la calidad de los pedidos. Por este motivo las compañías prestan especial atención a esta actividad, intensificando esfuerzos para optimizarla y lograr reducir costos y garantizar la satisfacción del cliente simultáneamente. El presente artículo pretende identificar las problemáticas del picking de empresas industriales y de distribución, así como establecer la relación de dichas dificultades con las técnicas de gestión y tecnologías disponibles. El método investigador utilizado ha sido el estudio Delphi.

Palabras clave: Sistemas de preparación de pedidos, gestión de almacenes, Estudio Delphi, picking

Abstract: Picking is a key factor in the warehouse administration due to its impact on the operation expenses, as well as its repercussion on orders quality. This is the reason why companies pay special attention to this activity, intensifying efforts to optimize it and trying to reduce costs and satisfy clients at the same time. This paper pretends to identify picking problems, as well as establish the relationship between these difficulties and the management techniques and technologies that might be used by industrial and distribution companies. The research method carried out in order to achieve this goal was a Delphi study.

Key words: Picking systems, Warehousing Management, Delphi study.

\section{Introducción}

El proceso de preparación de pedidos o picking es una de las actividades claves dentro del almacén debido al impacto que tiene sobre los costes de operación, pudiendo superar el $65 \%$ de los mismos (Coyle et al. 1996, De Koster et al. 2007) y sobre la calidad de los pedidos (De Koster et al. 2007).

El presente artículo identifica las problemáticas existentes en los sistemas de preparación de pedidos de empresas industriales y de distribución, así como establece la relación de dichas dificultades con las técnicas de gestión y tecnologías utilizables determinando un marco para su resolución. El método investigador empleado ha sido el estudio Delphi.

El documento se organiza de la manera puntualizada a continuación. La siguiente sección describe los diferentes sistemas de preparación de pedidos recopilados de la literatura más reciente. Las secciones 3 y 4 detallan los aspectos que en los últimos tiempos están incrementando la complejidad de la preparación de pedidos y las tecnologías utilizadas para llevar a cabo esta actividad de la manera más eficiente posible, respectivamente. La sección 5 incluye una breve descripción teórica del método empleado (Delphi), así como también del cuestionario en el que se han soportado las entrevistas a expertos empresariales durante el estudio de campo ejecutado. Finalmente, las secciones 6 y 7 desarrollan los resultados y las conclusiones alcanzadas una vez llevado a cabo el estudio, mientras que la sección 8 propone futuras líneas de investigación.

\section{Sistemas de preparación de pedidos}

La preparación de pedidos involucra las actividades de agrupación y planificación de las solicitudes de los clientes, el lanzamiento de la orden, la recolección de los artículos por sus respectivas localizaciones y el depósito de los mismos en el muelle de entrada y salida. (Coyle et al., 1996, Yan Shiau 2009)

Específicamente, las fases de la organización de la preparación de pedidos se pueden desglosar en preparación, desplazamientos, búsqueda, extracción y acondicionamiento. 
Una empresa que quiera disponer de un picking competitivo deberá coordinar tanto los elementos físicos, los métodos organizativos y las nuevas tecnologías para alcanzar un adecuado nivel de aprovechamiento de las instalaciones y de la productividad del personal (operatividad) y adicionalmente entregar en el plazo acordado, con la calidad de producto acordada y sin errores (calidad de servicio). Para medir estos conceptos de desempeño en los sistemas de preparación de pedidos Frazelle (2002) propone el sistema de indicadores detallado en la Figura I:

Figura 1

Tabla de indicadores en el proceso de picking. Frazelle, E (2002)

\begin{tabular}{|c|c|}
\hline Productividad & $\begin{array}{l}\text { Líneas de pedido/personas \& hora } \\
\text { Cajas o envases/persona \& hora }\end{array}$ \\
\hline Financiero & Coste/línea de pedido \\
\hline Utilización & $\begin{array}{l}\text { \% utilización del personal } \\
\text { \% utilización del equipamiento }\end{array}$ \\
\hline $\begin{array}{l}\text { Calidad del pedido } \\
\text { pedido }\end{array}$ & Exactitud del picking. Errores. \\
\hline $\begin{array}{l}\text { Cumplimentación } \\
\text { plazo }\end{array}$ & $\begin{array}{l}\text { Tiempo del ciclo de la } \\
\text { preparación }\end{array}$ \\
\hline
\end{tabular}

Existen varios factores de diseño de los sistemas de preparación de pedidos, los cuales se recogen en la Figura 2 y se describen a continuación:

Figura 2

Factores de diseño en sistemas de preparación de pedidos, diagrama de Goetschakckx and Ashayeri, 1989, modificado

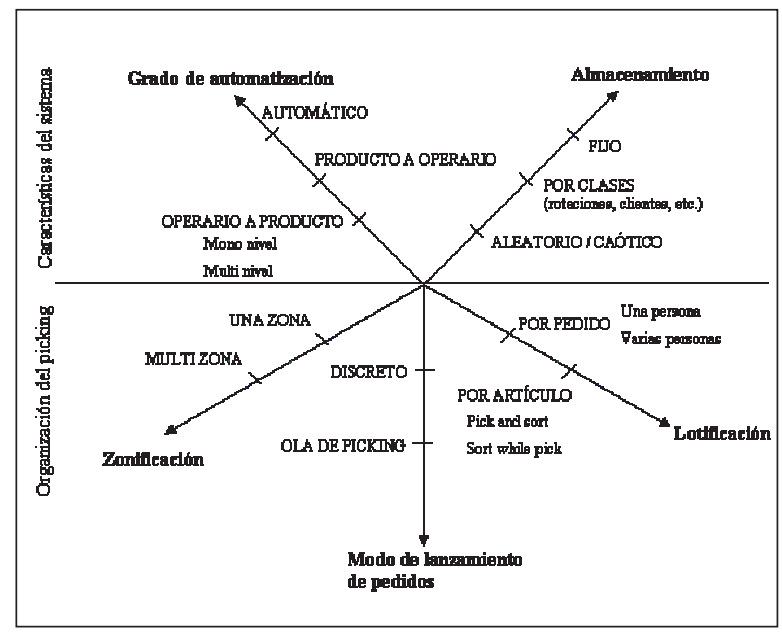

En primer lugar se puede hacer una división, en función del grado de automatización del proceso, en Sistemas Automáticos, Sistemas Producto a Operario y Sistemas Operario a Producto.
La característica principal de los sistemas del primer grupo es la ausencia de participación de personal en el picking. Estos sistemas son empleados en casos muy especiales (Rushton et al., 2006) debido al alto nivel de estandarización e inversión que la preparación requiere y no se han contemplado en el estudio realizado.

En los otros dos sistemas si existe la intervención humana, en mayor o menor grado. En el caso de una preparación Producto a Operario es la mercancía la que es trasladada hacia la localización del personal mediante sistemas de almacenamiento automático. En los sistemas Operario a Producto, en cambio, es la persona quien se desplaza a lo largo de los pasillos, a pie o haciendo uso de distintos equipos de manutención, con el objeto de recoger los materiales. (Manzini 2007).

Dentro de los sistemas Operario a Producto puede distinguirse a su vez una subclasificación según la altura del picking. Ésta se compone de Picking mono nivel (manual) y Picking multi nivel (en altura).

Otra característica del sistema de preparación de pedidos está vinculada al tipo de almacenamiento empleado. Los métodos de ubicación se clasifican en tres grupos de Asignación fija, Asignación por clases y Asignación caótica (Dekker et al., 2004) En el primero de los casos, los nichos o ubicaciones están identificados con el código y/o nombre de artículo de tal manera que una referencia siempre se coloca en el mismo lugar. En los otros dos sistemas, la asignación se realiza una vez recepcionado el producto. Los criterios para establecer la asignación por clases con los cuales se realiza esta ubicación pueden ser diversos, tales como asignación por familias de producto o por nivel de rotación o por clientes. En el tercer caso la asignación es aleatoria.

Por otra parte, es posible clasificar la preparación de pedidos según la organización de la misma Por pedido y Por artículo. Si el picking se realiza pedido a pedido se está en el primer caso. Por el contrario, si se realiza una agrupación o «batch» de pedidos se considera que la selección se hace por artículo (Petersen 2004, Mengfei y De Koster 2009). El primer caso puede ser realizado tanto por una única persona como por varias, no comenzando a preparar el siguiente pedido hasta no terminar el anterior. En el segundo caso, el operario que trabaja dentro de la zona de preparación realizando una extracción agrupada, pude realizar la clasificación en pedidos a medida que extrae productos de una ubicación (sort while pick) o puede esperar a finalizar todo el reco- 
rrido para realizar la clasificación (pick and sort) (Van Den Berg 1999, De Koster et al. 2007, Carrasco-Gallego y Ponce-Cueto 2008)

Figura 3.

Tipos de picking según intervención humana - Errasti, 2007

\begin{tabular}{|c|c|c|}
\hline & \multicolumn{2}{|c|}{ Tipo de picking } \\
\hline Número & $\begin{array}{c}\text { Una persona } \\
\text { Pedido a pedido }\end{array}$ & $\begin{array}{c}\text { Una persona } \\
\text { Extracción agrupada }\end{array}$ \\
\hline nas & $\begin{array}{l}\text { Varias personas } \\
\text { Pedido a pedido }\end{array}$ & $\begin{array}{c}\text { Varias personas } \\
\text { Extracción agrupada }\end{array}$ \\
\hline
\end{tabular}

Otra forma de clasificación puede realizarse en función del modo de lanzamiento de órdenes de pedido en Discreto y Ola de pedido (wave picking) El término ola de pedido es utilizado cuando órdenes con destinos comunes son lanzadas simultáneamente. (De Koster et al. 2007).

Finalmente, la preparación de pedidos puede ser dividida en función de la zonificación del almacén en Una zona y Multi zona. Si existe una zona única para el almacenamiento de los productos, se está en el primer caso, mientras que, si el depósito está dividido en distintas áreas a las cuales se debe acceder para cumplimentar el pedido se está en presencia de la segunda forma de picking.

\section{Complejidad del picking}

Algunos autores (Van Den Berg 1999, Rushton et al. 2006, De Koster et al. 2007), afirman que en los últimos años se ha dado un incremento en la complejidad a la hora de realizar la preparación de pedidos. A continuación se detallan algunas variables, así como las causas que contribuyen a dicho fenómeno:

\section{I. Aumento en el número de referencias}

Debido a las estrategias de diversificación que desarrollan las empresas con el objeto de impulsar su nivel competitivo, ha habido un incremento en el número de referencias a almacenar. Este hecho deriva en un aumento en el volumen de pedidos y en las líneas de los mismos, así como en una mayor heterogeneidad de tipos de pedidos.

\subsection{Aumento del número de pedidos junto con la reducción de cantidades por pedido}

Las empresas buscan un mayor grado de eficiencia a través de la reducción de los niveles de stock, y como consecuencia se ha constatado en los almacenes aguas arriba a dichas empresas un incremento en el número de pedidos a preparar, así como una reducción en las cantidades a suministrar por pedido. Esto hace que el volumen de preparación al que deben hacer frente los centros de almacenaje se haya incrementado considerablemente en los últimos años, originando un aumento en los costes de preparación por volumen total suministrado. Por otra parte, el fenómeno mencionado ha supuesto un incremento de la frecuencia de entregas y preparaciones para un mismo cliente, lo cual ha originado una disminución en los ciclos de pedido a preparar.

\subsection{Mayor nivel de personalización de los pedidos}

Debido a las mayores exigencias de clientes en relación a la personalización de los pedidos, los centros de almacenaje deben realizar un mayor número de operaciones de manipulación y acondicionamiento de los productos extraídos.

\subsection{Reducción en unidades mínimas de servicio}

A raíz de la disminución de cantidades por pedido, en los últimos años se ha observado una reducción en las unidades mínimas de servicio establecidas por las empresas. Estas unidades han pasado de ser pallets completos a ser en su mayoría cajas e incluso unidades sueltas de producto. Esto trae aparejado un aumento en los movimientos a realizar en la preparación de pedidos, lo que supone un tiempo mayor a ser destinado a dicha actividad.

\section{Tecnologías disponibles en la preparación de pedidos}

El empleo de la informática y las nuevas tecnologías permiten mejorar los niveles de eficacia y eficiencia. Este uso se refleja, entre otros beneficios, en el la eliminación de errores y en la reducción de los costos operativos. A continuación se describen distintas tecnologías utilizables actualmente en los almacenes: 


\section{I. Gestión de ubicaciones informatizada}

La gestión de ubicaciones hace referencia a la asignación de un nicho o hueco a un artículo de tal manera que se conocen los productos almacenados y donde están ubicados. Estos productos van a ser extraídos posteriormente en el proceso de picking, y es por ello que una adecuada gestión de ubicaciones puede permitir un sistema más eficiente.

\subsection{Sistema de código de barras}

El empleo de los sistemas de código de barras para la codificación del producto e incluso de las ubicaciones permite en el proceso de preparación de pedidos asegurar la coincidencia entre el pedido emitido por el cliente y los artículos enviados al mismo, evitando los errores de sustitución, omisión e inclusión. En las empresas el empleo de los lectores de códigos de identificación de artículos suele ir acompañado por terminales móviles que permiten su conectividad con los sistemas de gestión de los almacenes.

\subsection{Terminales móviles y radiofrecuencia}

Los terminales móviles, PDAs y/o ordenador personal que emplean los operarios para realizar el picking están conectados al sistema de gestión del almacén por medio de sistemas de radiofrecuencia. Dicho sistema cubre el área entera del almacén y permite a todos los terminales móviles comunicarse con el sistema de gestión de pedidos. Debido a la conexión en línea a cada terminal, el sistema de gestión del almacén puede asignar la recogida óptima al preparador en función con su posición dentro del almacén, permitiendo la minimización del número de viajes en vacío y el aumento de la eficiencia.

\subsection{Pick to light}

En este tipo de picking las instrucciones de extracción se comunican al operario a través de indicadores luminosos que señalan de dónde se deben extraer los productos y la cantidad a extraer. Esta tecnología ayuda a identificar inmediatamente la mercancía reduciendo los tiempos de búsqueda del preparador (Rushton et al., 2006)

\subsection{Pick to voice}

El sistema pick to voice es una variante de recogida de productos en la cual los operarios son dirigidos a las distintas ubicaciones a extraer el producto mediante la utilización de auriculares conectados a la terminal de datos (Rushton et al., 2006).

\subsection{Báscula}

Una vez realizada la extracción de todas las referencias de un pedido, uno de los sistemas que se emplean para detectar errores de conteo o errores de elección (omisión o inclusión) es el de la báscula. A través de ella se pesan los artículos extraídos y el sistema compara su peso real con el que teóricamente debiera tener el pedido.

\section{Metodología}

El estudio Delphi, desarrollado por Helmer y Dalkney (1975) consiste en un proceso interactivo, a partir de soluciones aproximadas, en el cual se refuerza la validez de los resultados obtenidos mediante la comunicación entre el investigador y los expertos involucrados en el mismo (Turoff 2006). Inicialmente se realizó un cuestionario (Ver Anexo) en función de la información obtenida en la revisión bibliográfica. Luego, se contactó con diversos expertos y se realizó la entrevista. Finalmente, al obtenerse los resultados, se volvió a consultar a los especialistas logrando la aceptación definitiva del estudio.

Se escogieron como expertos, directivos logísticos y jefes de almacén con conocimientos relacionados a problemáticas de preparación de pedidos con un cierto grado de complejidad.

El criterio de complejidad empleado para seleccionar a los expertos empresariales para la realización de las entrevistas se basó en la posesión de: Más de 500 referencias almacenadas, Más de 100 pedidos al día, Más de 500 líneas por pedido al día.

El estudio se centró sobre almacenes localizados principalmente en el entorno geográfico del Norte de España. Finalmente, 4 I empresas de una población de 250 con almacenes con preparación de pedidos para empresas industriales y de distribución con las características mencionadas, fueron analizadas. Según Akkermans (2002), al contar con un grupo superior a 20 especialistas se minimiza el riesgo de que las opiniones particulares contaminen el resultado agregado. Fue debido a este hecho, y a la diversidad de sectores estudiados, que la muestra de empresas se considera representativa. 
En la Figura 4 se pueden observar los distintos sectores a los cuales pertenecen las empresas y personas contactadas:

Figura 4.

Distribución de los sectores de las empresas entrevistadas

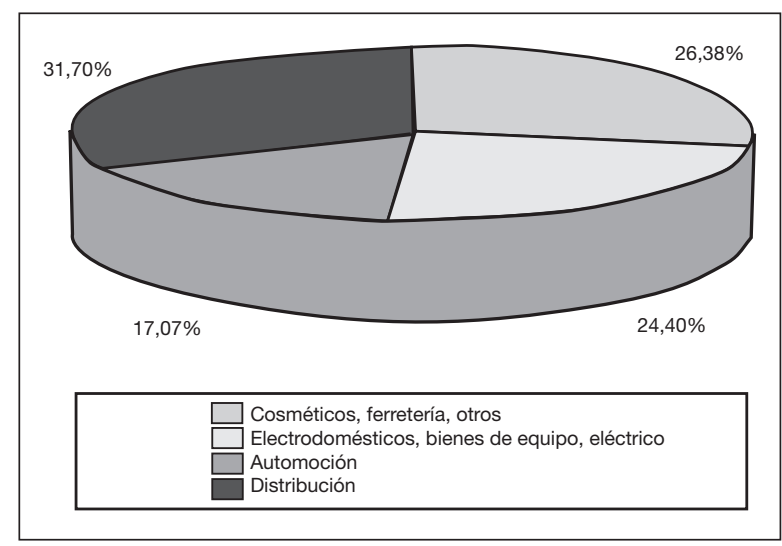

Por otra parte, se ha buscado también la segmentación respecto a la localización dentro de la cadena de suministro. En la Figura 5 se puede observar la localización de las empresas entrevistadas en la cadena de suministro correspondiente al sector al cual pertenecen:

Figura 5

Número de empresas entrevistadas según la posición en la cadena de suministro

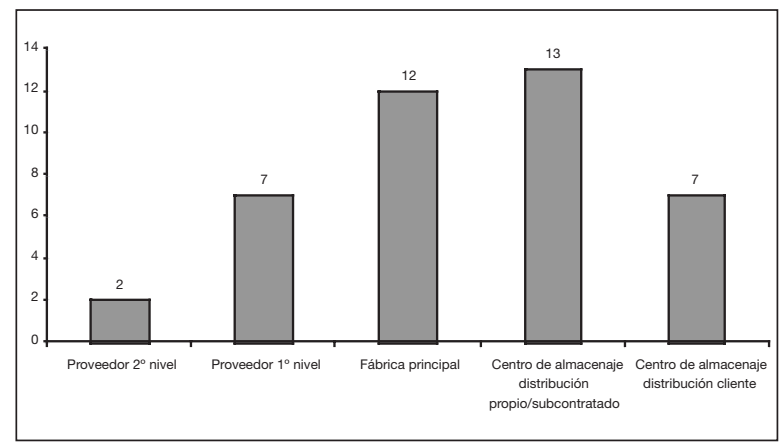

Adicionalmente, del total de empresas, el 48.8\% han sido entrevistas relacionadas con sistemas de preparación de pedidos en centros de almacenaje de producto terminado mientras que el $51.2 \%$ han sido almacenes de materias primas y componentes.

Para facilitar la labor del estudio Delphi, se desarrolló un cuestionario que permitió estratificar los resultados según localización en la cadena de suministro y sector, comprobar el aumento de la complejidad de la preparación de pedidos (Ver apartado 3), conocer el rendimiento de los sistemas de preparación según indicadores de rendimiento (Ver figura I) y las técnicas organizativas (Ver apartado 2) y tecnologías empleadas (Ver apartado 4).

\section{Resultados}

En relación a los puntos detallados en el Apartado 3 Complejidad de picking, prácticamente el $98 \%$ de los centros de almacenaje (tanto de materias primas y componentes, como de distribución) analizados tienen que hacer frente a este incremento en el número de referencias.

El aumento del número de pedidos junto con la reducción de cantidades por pedido ha sido señalado por 3 de cada 4 empresas entrevistadas. Especialmente reseñable resulta este aspecto tanto en aquellas empresas enmarcadas en sectores tales como el de automoción, así como centros de almacenaje de distribución cercanos al cliente final. Un 60\% de empresas constatan que las actividades de acondicionamiento y personalización de pedidos han aumentado.

En cuanto al rendimiento se ha constatado que la gran mayoría miden el rendimiento de sus sistemas de prelación de pedidos según los indicadores propuestos por Frazelle (2002), así como que en ciertos sectores, principalmente en almacenes de distribución de gran consumo y alguno de bienes industriales, se ha alcanzado un nivel de calidad de pedidos cercano a la excelencia.

En relación a los procesos operativos o fases asociados al picking que se distinguen en el Apartado 2 Sistemas de preparación de pedidos, se han obtenido los \% de tiempo dedicados a cada una de ellas. Con respecto a las actividades de acondicionamientos, el cada vez mayor nivel de personalización de los pedidos hace que sean más las operaciones necesarias al final del proceso.

Se constató que el $74 \%$ de las empresas entrevistadas emplean sistemas Operario a Producto en el proceso de preparación. Si se clasifica la información obtenida según el sistema de picking empleado, la dedicación a cada fase del picking es distinta. En la Figura 13 se pueden observar dichas diferencias entre los sistemas Operario a Producto y los sistemas Producto a Operario respectivamente.

Son las operaciones de desplazamiento y acondicionamiento las que mayor porcentaje de tiempo suponen en los sistemas Operario a Producto. En aquellas empresas que tienen, en su mayoría, sistemas 
Figura 13

Tiempos dedicados a las fases del picking. Comparación Operario a Producto y Producto a Operario

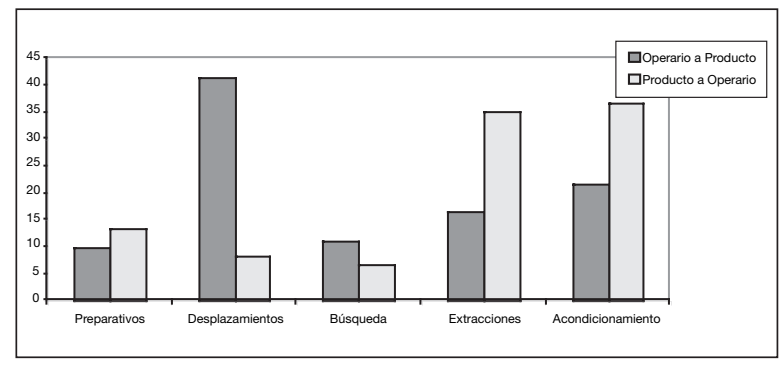

Producto a Operario, las actividades de desplazamiento pasan a un segundo plano, siendo las actividades de acondicionamiento y extracción las más intensivas en tiempo. Esto se debe a que la automatización de los sistemas de almacenaje provoca una disminución del tiempo total de la preparación de pedidos, eliminando el tiempo de búsqueda y desplazamiento de los operarios.

Por otra parte, se comprobó que el picking supone, en promedio, un 58,5\% del total de costes de almacén.

Adicionalmente se constató que la preparación de pedidos es la actividad con mayor intensidad en personal en almacenes con sistemas manuales (sistemas Operario a Producto) y con mayor intensidad en capital en almacenes con sistemas automatizados (sistemas Producto a Operario y sistemas picking sin intervención humana). En el primer caso, el porcentaje de coste incurrido por concepto Mano de Obra, tanto directa como indirecta, respecto al total de costes de almacén supone $64,3 \%$, mientras que este porcentaje desciende a un $26,7 \%$ en almacenes con sistemas Producto a Operario.

Con respecto a las tecnologías empleadas (gestión de ubicaciones informatizadas, código de barras, terminales móviles y radio frecuencia, pick to Light, put to voice, sistemas producto a operario, sistemas operario a producto mononivel, sistemas operario a producto multinivel), se relacionaron las mismas con la complejidad en la preparación de pedidos (número de referencias, número de pedidos y cantidad por pedido, personalización de pedidos, unidedas mínimas de servicio), las técnicas organizativas empleadas (notificación por pedido o por artículo, zonificación y lanzamiento de pedidos) y la posición en la cadena logística de las empresas consideradas en el estudio, entre otros factores. Los resultados conseguidos se resumen en la matriz de la Figura 14.

\section{Conclusiones}

- Existen diferentes sistemas de preparación de pedidos, y la elección de implantar uno u otro depende de que la complejidad de la preparación de pedidos sea de un nivel bajo, medio o alto (Ver figura 14). Las empresas con mayores niveles de exigencia de calidad de servicio y mayores volúmenes de líneas de pedido a preparar emplean las soluciones tanto técnicas como tecnológicas más avanzadas, así como métodos de organización del picking más complejos. Estas problemáticas se han identificado en algunos centros de almacenaje de producto terminado de empresas de distribución.

- Las empresas de complejidad media de preparación de pedidos para mejorar su competitividad emplean técnicas organizativas y tecnologías asociadas en sistemas Operario->Producto que les permitan mantener niveles de eficiencia productiva altos. Sin embargo se ha constatado que algunas comienzan a implantar sistemas de almacenamiento y preparación avanzados con Sistemas Producto->operario.

- La gran mayoría de los centros de almacenaje están afrontando la necesidad de responder a un incremento de la complejidad de la preparación de pedidos en aumento del número de referencias, tipologías de clientes pedidos, nivel de personalización de pedidos y reducción de unidades servidas en ciclos de respuesta más cortos.

- Se ha constatado que más de la mitad de los costos operativos totales del almacén son producto de las actividades de preparación de pedidos. Por este motivo, las empresas intensifican esfuerzos buscando optimizar el proceso, invirtiendo en tecnologías que disminuyan errores y agilicen las operaciones. La utilización de sistemas producto a operario reduce el peso relativo de los tiempos de desplazamiento desde un $41.3 \%$ en el caso de sistemas Operario a Producto, a un 8.3\% en sistemas Producto a Operario. Este fenómeno permite una mayor dedicación a las actividades de acondicionamiento y extracción, reduciendo errores a la hora de la preparación de los pedidos.

\section{Líneas futuras}

Rowley afirma que el Estudio de Casos o Case Studies es válido para el propósito de testeo de teorías. Otros autores [Yin, 1990], añaden que el Estudio de Casos, puede ser utilizado para distintos tipos de 


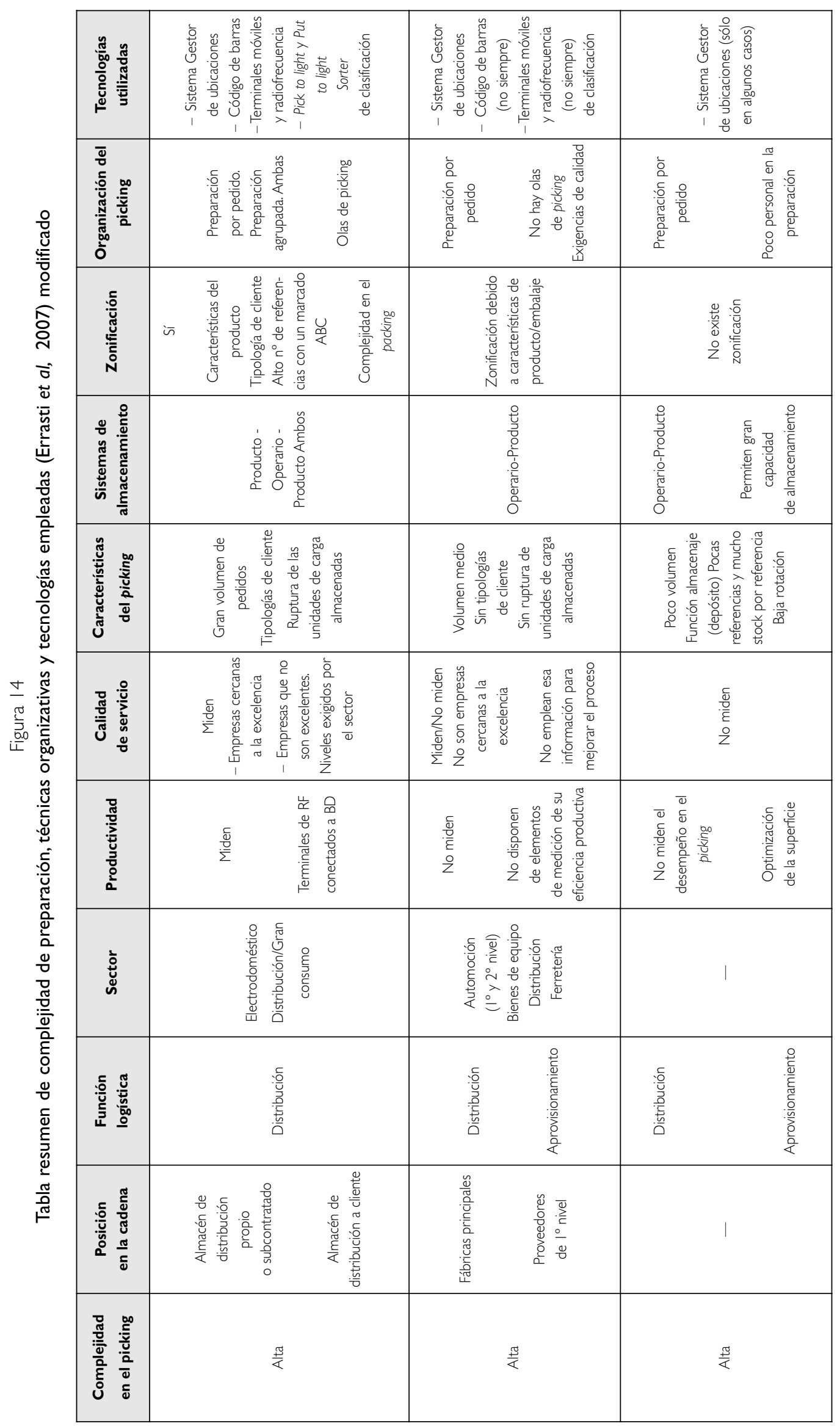


propósitos de investigación como prospectivas, construcción de nuevas teorías, testeo de teorías y extensión o afino de teorías existentes [Yin, 1990].

Por tanto, tras la creación del marco de análisis que relaciona la complejidad de la preparación de pedidos, con las técnicas organizativas y tecnologías utilizables para el rediseño de sistemas de preparación de pedidos, se proponen desarrollar Estudios de Casos para profundizar en la creación de nuevas teorías y modelos para la mejora y optimización de los sistemas de preparación de pedidos.

De las aportaciones realizadas en el Estudio Delphi dichas líneas futuras podrían ser:

Un estudio sobre los sistemas de almacenamiento y preparación de pedidos Producto-Operario, su impacto sobre el desempeño logístico y retorno de inversión.

La aplicación de metodologías de rediseño de sistemas de planificación operativo (GRAI, D.B.R.,..) ampliamente estudiadas en sistemas productivos, con un alto grado de aplicación en los sistemas de preparación de pedidos más complejos de grandes plataformas de distribución.

La creación de un nuevo marco de trabajo «100\% Pedidos Perfectos» orientados a la búsqueda del concepto «0 Defectos» en almacén. Esto permitiría desarrollar la mejora de la calidad de proceso en los procesos de almacén. (Errasti 2007)

\section{Referencias Bibliográficas}

AKKERMANS HENK, A., BOGERD PAUL, YUCESAN ENVER ANDVANWASSENHOVE LUK, N. (2003). The impact of ERP on supply chain management: Exploratory findings from a European Delphi study, European Journal of Operational Research, Volume 146, Issue 2, Pages 284-301,

BLACKSTONE, J. and COX, F. (2004). APICS Dictionary: I I th Edition, CFPIM, CIRM, Alexandria.

CARRASCO-GALLEGO RUTH y PONCE-CUETO EVA (2008). Mejora de la eficiencia de una central logística mediante el resideño del reaprovisionamiento de la zona de Picking (Improving the efficiency of a logistics center by redesigning piece picking area replenishment), Dirección y Organización, Núm. 36, ISSN: I I 32- I75X.

COYLE, J.; BARDI, EJ.; LANGLEY CJ. ( 1996). The management of business logistics, $6^{\text {th }}$ edition, West Publishing.

DE KOSTER, R.; LE-DUC,T.; ROODBERGEN, J. (2007). Design and control of warehouse order picking: A litera- ture review. European Journal of Operational Research |02, 48|-50|.

DEKKER, R.; KOSTER, M.B.M.; ROODBERGEN, K.J. y KALLEVEEN, H. (2004). «Improving Order-Picking Response Time at Ankor's Warehouse». Interfaces, 34, 4, pp. 303313.

ERRASTI, A. (2007). Marco de análisis de alternativas de sistemas de preparación de pedidos, aspectos a valorar en la reingeniería de procesos de almacén, Primer Congreso de Logística y Gestión de la Cadena de Suministro, Zaragoza.

ERRASTI, A. (2007). Proyecto OPP Optimización Preparación de Pedidos, Cluster de Transporte y Logística de Euskadi.

ERRASTI, A. (2006). KATAIA: Modelo para el análisis y despliegue de la estrategia logística y productiva, Doctorado Tecnun, San Sebastian.

FRAZELLE, E. (2002). World-Class Warehousing and Material Handling, McGraw-Hill, New York.

GOETSCHALCKX, M., ASHAYERI, J. (1989). Classification and design of order picking systems. Logistics World, 99-106.

JIUN-YAN SHIAU, A., MING-CHANG LEE, BC. (2009). A warehouse management system with sequential picking for multi-container deliveries.

MANZINI, R.; GAMBERI, M.; PERSONA, A.; REGATTIERI, A. (2007). Design of a class based storage picker to product order picking system. International Journal of Advanced Manufacturing technologies 32, 81 1-821.

MAULEON, M. (2003). Sistemas de almacenaje y picking. Ediciones Diaz de Santos, Madrid.

MENGFEIYU, RENE M. B. M. DE KOSTER (2009). The impact of order batching and picking area zoning on order picking system performance. European Journal of Operational Research 198(2): 480-490.

PERTERSEN CHARLES G., AASE GERARD (2004). A comparison of Picking, storage, and routing polices in manual order picking, International Journal of production economics 92, I1 - 19 .

ROWLEY, J. (2002). Using Case Studies in Research.Management. Research News Vol $25 \mathrm{~N} \mathrm{I}$.

RUSHTON, A.; CROUCHER, P. \& BAKER, P. (2006). The Handbook of Logistics and Distribution Management, Kogan Page, London.

TUROFF, M. and HILTZ, SR. Computer based Delphi Processes: http://web.njit.edu/ turoff/Papers/delphi3.html

VAN DEN BERG, J. P. (1999) A literature survey on planning and control of warehousing systems. IIE Transactions, 31, 75I-762.

YIN, R.K. (1990). Case Study Research: Design and Methods. Sage Publications, Beverly Hills. 